\title{
Overview of PIV in Supersonic Flows
}

\author{
Fulvio Scarano
}

Aerospace Engineering Department, Delft University of Technology, The Netherlands

f.scarano@tudelft.nl

\begin{abstract}
The extension of particle image velocimetry to supersonic and hypersonic wind-tunnel flows has been achieved in the last decade. This was mainly possible with the advent of short interframing-time CCD cameras with temporal resolution allowing to obtain correlated particle images at flow velocities exceeding $1000 \mathrm{~m} / \mathrm{s}$. The most challenging aspects of PIV experiments in supersonic flows are still recognized as the seeding-particle-selection and seeding-distribution techniques. Also, the optical access for illumination and imaging require a specific attention since pressurized facilities offer limited optical access. The presence of shock waves in supersonic flows introduces regions where particle tracers slip with respect to the surrounding flow. Moreover, the particle seeding density becomes strongly nonuniform and particle-image blur can occur as a result of the strong refractive index variations. The present chapter reviews the physical and technical problems of PIV experiments and discusses the potential of such techniques on the basis of recent experiments performed in high-speed wind tunnels: double compression ramp at Mach 7 and shock-wave turbulent boundary interaction at Mach 2.
\end{abstract}

\section{Introduction}

The technological relevance of compressible flows in both scientific and industrial research is well acknowledged, since it constitutes an established branch of science and a field of ongoing research and development for industrial, mostly aerospace, applications. Experimental high-speed investigations rely as a rule on wind-tunnel facilities producing a controlled uniform high-speed stream in which the model under investigation is immersed. Once the first step of realizing controlled test conditions is achieved, a second one of equal importance must be accomplished, consisting of the actual measurement of the relevant flow quantities. Among the techniques that can be employed for supersonic flow measurements, nonintrusive optically based techniques have received large attention due to their promising performances. Starting from qualitative techniques such as shadowgraphy and Schlieren methods [1], the experimental community has developed quantitative methods such as holographic interferometry [2,3], laser Doppler velocimetry [4, 5], laser-induced fluorescence [6, 7] and particle image velocimetry [8]. The present chapter gives a detailed discussion about the current status of PIV capabilities in supersonic and hypersonic wind-tunnel flows.

\footnotetext{
A. Schroeder, C. E. Willert (Eds.): Particle Image Velocimetry,

Topics Appl. Physics 112, 445-463 (2008)

(C) Springer-Verlag Berlin Heidelberg 2008
} 
Supersonic flows are characterized shock by waves that challenge measurement techniques, where a large flow deceleration occurs across a very thin region. Also, boundary layers and shear layers can be relatively thin due to the limited turbulent mixing typical of the compressible regime [9] and the high values of the Reynolds number for high-speed flows. Flow compressibility introduces two additional difficulties, namely the large spatial variation of the particle-seeding density and the inhomogeneous refractive index throughout the flow field. The latter results in a distortion of light propagation for optically based measurement techniques [10].

The application of PIV in compressible flows was pioneered by Moraitis and Riethmuller [11] and Kompenhans and Höcker [12] who used the photographic recording technique combined with image-shifting methods. The advent of frame-straddling CCD cameras and short-duration nanosecondpulsed double-cavity Nd:YAG lasers turned the technique into a versatile tool finally operated not only by the developers' research groups. Investigations range from supersonic jets $[13,14]$ to transonic turbomachinery flows $[15,16]$. Turbulence measurements in compressible mixing layers were more recently performed with PIV by [17]. Supersonic turbulent wakes as well as shockwave turbulent boundary-layer interaction were investigated by the author and coworkers [18-21]. The technique was first extended to the hypersonic flow regime by Haertig et al. [22] at ISL and further by Schrijer et al. [23] at TU Delft.

\section{Flow Seeding and Imaging}

The main requirements for the flow seeding particles is that they provide an accurate flow tracing still at a sufficient particle-seeding density. The flow-tracing capability of particles of diameter $d_{\mathrm{p}}$ and a specific gravity $\rho_{\mathrm{p}}$ is usually quantified through the particle relaxation time $\tau_{\mathrm{p}}$. The theoretical behavior for small spherical particles may be reduced to the modified Stokes drag law [24]. Given the relatively low value of the Mach number and Reynolds number based on the particle diameter, the modified drag relation that takes into account rarefaction effects yields the following expression for the relaxation time

$$
\tau_{\mathrm{p}}=d_{\mathrm{p}}^{2} \frac{\rho_{\mathrm{p}}}{18 \mu_{\mathrm{f}}}\left(1+2.7 \mathrm{Kn}_{\mathrm{d}}\right)
$$

where $\mathrm{Kn}_{\mathrm{d}}$ is the Knudsen number obtained as the ratio between the molecular mean free path and the diameter of the particle tracers $d_{\mathrm{p}}$. A detailed study on the particle motion in supersonic flows is provided by [25].

In several flow conditions the Knudsen number is a function of the particle Reynolds number and relative Mach number between the particle and the surrounding fluid $M_{\Delta V}$, following the relation $\mathrm{Kn}_{\mathrm{d}}=1.26 \sqrt{\gamma} M_{\Delta V} / \mathrm{Re}_{\mathrm{d}}$, [26] 
where $\gamma$ is the gas specific heat ratio $(\gamma=1.4$ for air). The Reynolds number of the flow around the slipping particles is

$$
\operatorname{Re}_{\mathrm{d}}=\frac{\rho_{\mathrm{f}} \cdot \Delta V \cdot d_{\mathrm{p}}}{\mu_{\mathrm{f}}},
$$

where $\rho_{\mathrm{f}}$ and $\mu_{\mathrm{f}}$ are the fluid density and dynamic viscosity, respectively. The flow-tracing accuracy within a given experiment is then related to the Stokes number $S_{\mathrm{k}}$ expressing the ratio between $\tau_{\mathrm{p}}$ and the characteristic flow timescale $\tau_{f}$. Recalling that the most critical conditions are always encountered across shocks where $S_{\mathrm{k}} \gg 1$, the most critical conditions in turbulent flows are met when particle tracers are immersed in thin shear layers, for instance downstream of sharp separation points [27]. Samimy and Lele [28] suggested the following expression for the flow timescale in a free shear layer of thickness $\delta$

$$
\tau_{\mathrm{f}}=10 \cdot \frac{\delta}{\Delta V}
$$

and performed a particle dynamics computation associating the RMS slip velocity with the Stokes number. In conclusion, a Stokes number of 0.1 corresponds to a RMS slip velocity of approximately $1 \%$, which may be considered as an acceptable error in view of the other sources of error associated with the measurement technique.

\section{Experimental Assessment of Particle Response}

The measurement of the particle response time with PIV is technically challenging for numerous reasons. In many cases, the particle properties cannot be directly evaluated: scanning electron microscopy of solid particles only yields the particle properties when lying on a physical support and particle agglomeration introduces an uncertainty on the effective particle size when introduced in the flow [29]. Moreover, the effective particle density can only be predicted with a rough approximation for porous seeding materials. Liquid droplets may undergo condensation-evaporation from their production to the measurement section, which affects the particle size. It is therefore best practice to infer the dynamic particle response through a well-defined input flow test case. A planar oblique shock wave constitutes the commonly adopted test, which corresponds to a sharp velocity falling edge, through which particle tracers decelerate gradually due to inertia. Such a procedure was adopted in previous investigations $[4,17,18,24,30]$. The particle relaxation time $\tau_{\mathrm{p}}$ is then evaluated from the measurement of the relaxation length/time to the flow velocity behind the shock wave. It should be said, however, that the particle-velocity measurement across a shock wave may often be inaccurate due to the combined effect of numerous sources of error; the most important being: 




Fig. 1. PIV recording across a shock wave. Blur is visible at location $B$ between the dashed lines

1. Nonmonodispersed particle diameter distribution introduces a different velocity lag for neighboring particles traveling even in the same region of space. This leads to a broadening of the velocity correlation peak or even to incorrect velocity measurements. Moreover, in actual experiments the relatively large size of PIV interrogation windows often covers the entire relaxation length [30] and introduces two peaks in the correlation map corresponding to the particle velocity upstream and downstream of the shock, respectively;

2. Finite time separation between laser pulses. In high-speed flows the temporal resolution is often limited by the minimum interframing time that is of the same order as $\tau_{\mathrm{p}}$. This will result in a smearing of the velocity distribution in proximity of the shock position. Turbulence in the flow free stream causes small unsteady fluctuations in the position of the shock. Ensemble-averaged measurements will be smeared by such an effect.

3. Optical distortion due to nonuniform index of refraction (spatial blur) may introduce an important bias error on the velocity and an RMS error due to particle image blur as investigated by [31]. Figure 1 shows the blurring effect encountered when measuring the flow across shock waves with the viewing direction aligned with the planar shock. The cross-correlation peak will not only broaden across the shock region, increasing the measurement uncertainty, but may also skew (Figs. 2 and 3), which causes a bias error as shown in Fig. 4. These effects can be reduced by arranging the viewing from the upstream side of the shock of a few degrees.

Despite the above-mentioned effects, several PIV experiments could be conducted, which were able to infer the particle-tracer response. For instance, in the situations depicted below the two-dimensional flow across shock waves measured by PIV returned a response time of approximately two microseconds with sub-micrometer sized titanium dioxide particles.

\section{Online Seeding in Supersonic Wind Tunnel}

The transonic-supersonic wind tunnel (TST) of the Aerodynamics Laboratories at Delft University of Technology is a blowdown-type facility generating flows in a Mach range from 0.5 to 4.2 . The test section area is 

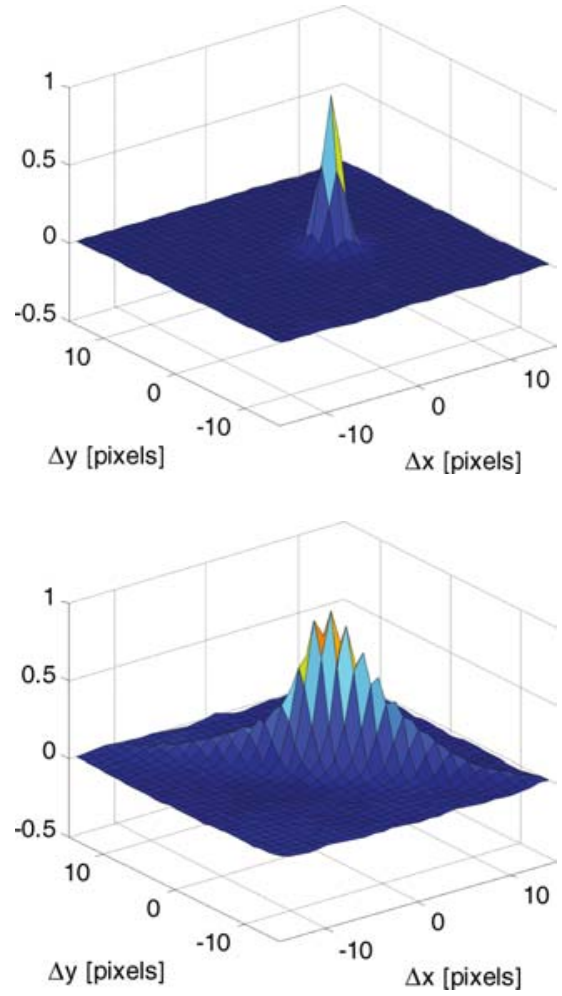

Fig. 2. Image crosscorrelation map of undistorted particle images at location $A$

Fig. 3. Image crosscorrelation map of skewed particle images at location $B$

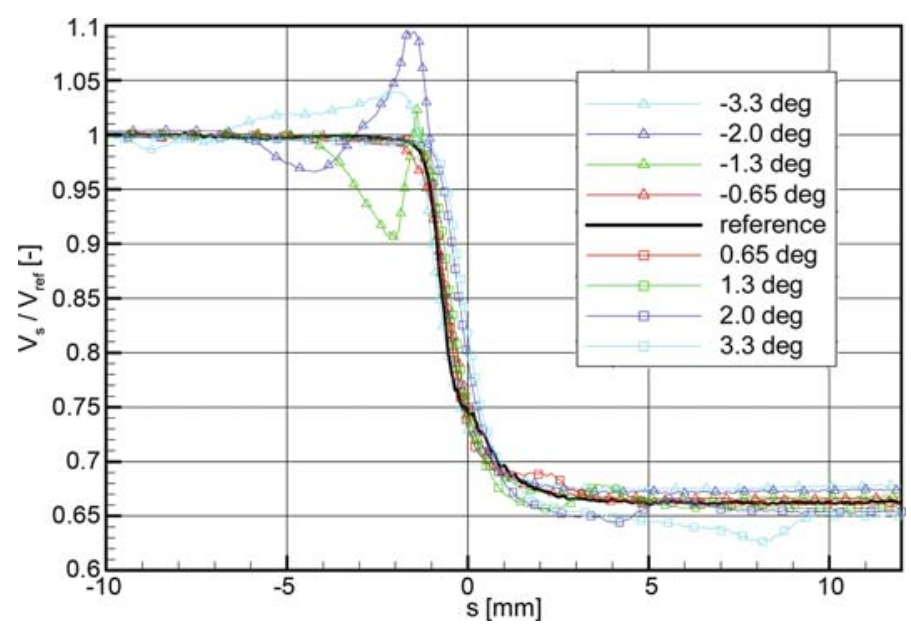

Fig. 4. Velocity measurements across the shock from different viewing angles (wrt shock plane, positive values indicate view from upstream of the shock) 


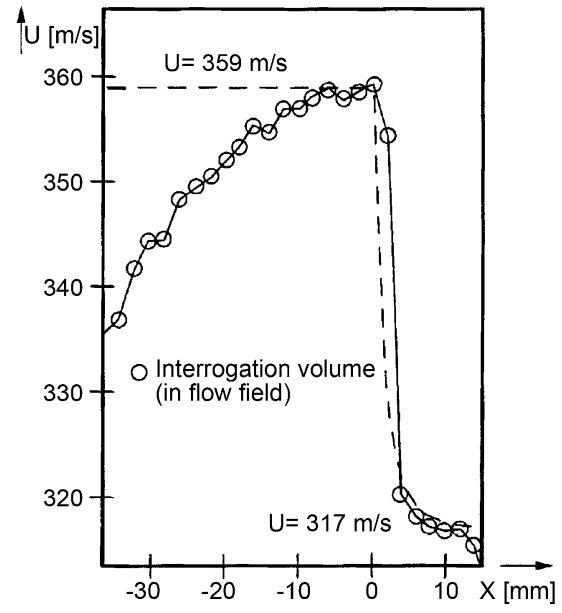

Fig. 5. PIV measurements, $\left(d_{\mathrm{p}}=\right.$ $1.7 \mu \mathrm{m})$ and theory (dashed line). Flow across a shock produced by a bluff cylinder [30]

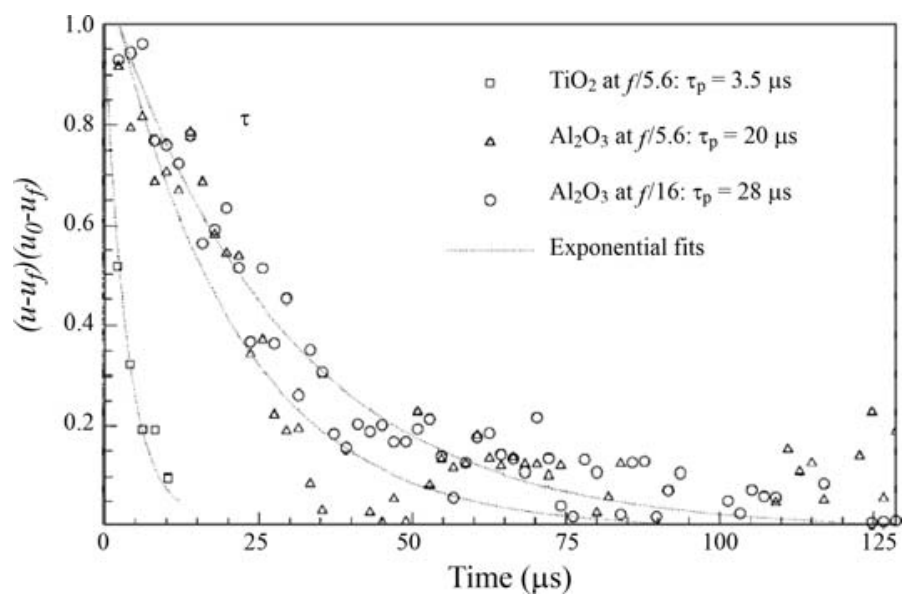

Fig. 6. Particle relaxation traces from different particle size and material. Oblique shock wave at $M=1.8$, deflection $\theta=10 \mathrm{deg}$ [17]

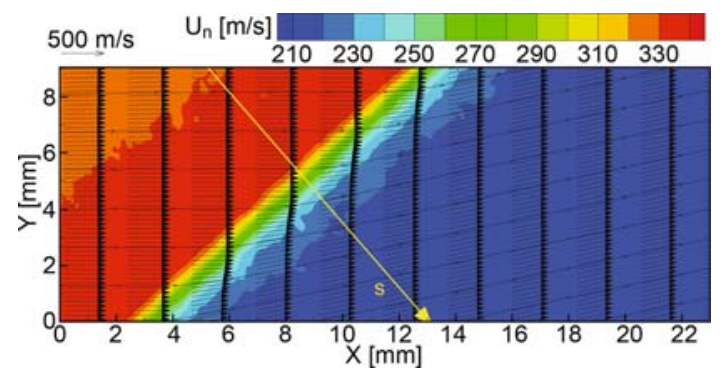

Fig. 7. Mean velocity field across oblique shock wave $(M=2$, deflection $\theta=$ $11.3 \mathrm{deg})$. Normal velocity component contours. In yellow is the shock-normal abscissa [18] 


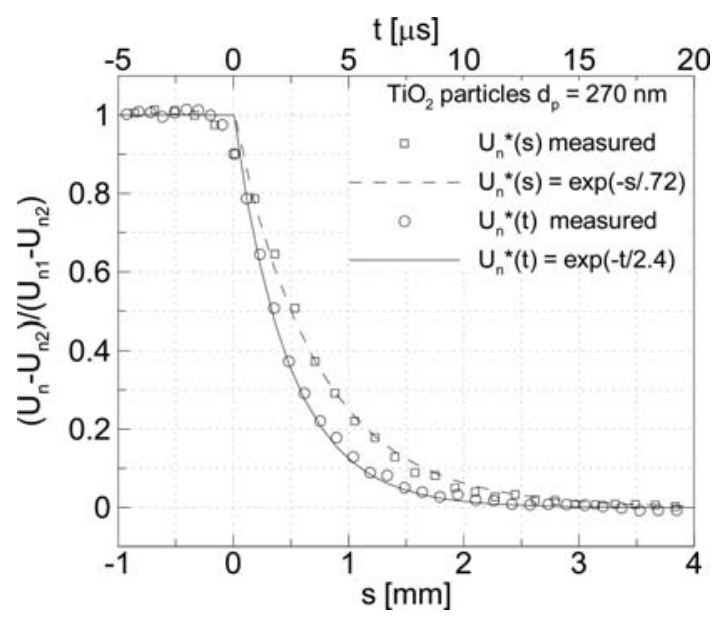

Fig. 8. Normalized velocity profile along abscissa $s$ from Fig. 7

$300(\mathrm{~W}) \times 270(\mathrm{H}) \mathrm{mm}^{2}$ and operates at values of the unit Reynolds number ranging from $38 \times 10^{6}$ to $130 \times 10^{6} \mathrm{~m}^{-1}$ with a typical model length of $10 \mathrm{~cm}$. In the transonic and supersonic ranges, the Mach number is set by means of a continuous variation of the throat section and flexible upper and lower nozzle walls. The wind-tunnel run time is about $300 \mathrm{~s}$. A fraction of the flow is seeded online in the settling chamber by means of a seeding distributor array one third the size of the settling chamber. The seeded flow within a streamtube allows PIV measurement in the test section over a region of approximately $10 \mathrm{~cm}$ diameter (Fig. 9). Under operating conditions, the seeded flow exhibits a mean particle concentration of about 10 particles $/ \mathrm{mm}^{3}$ estimated from PIV recordings. The interference of the seeding device onto the free-stream turbulence assessed with hot-wire anemometry (HWA) returned a barely detectable turbulence intensity increase $(0.2 \%)$ with a background turbulence of $1 \%$.

The particle-laden flow is produced by entraining titanium dioxide $\left(\mathrm{TiO}_{2}\right)$ particles with a high-pressure cyclone separator operated at 10 bar. The particle median diameter estimated by electron microscopy is $d_{\mathrm{p}}=0.4 \mu \mathrm{m}$, with a specific gravity estimated at $\rho_{\mathrm{p}}=1.0 \times 10^{3} \mathrm{~kg} \cdot \mathrm{m}^{-3}$. An example of the seeding conditions achieved in the TST-27 facility is shown in Fig. 14. Not all the flow is seeded and the interface of the seeded stream tube is clearly turbulent, which introduces undesired seeding intermittency for some cases.

\section{Storage-Tube Seeding in Hypersonic Wind Tunnels}

At higher Mach number in short-duration facilities (run time of milliseconds) the seeding technique becomes paradoxically more straightforward. This is 


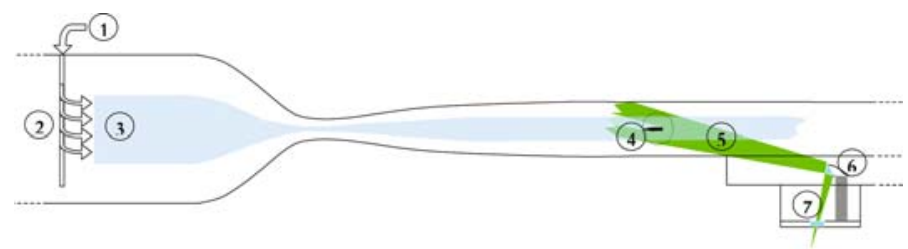

Fig. 9. Schematic of the seeding distribution device and lightsheet delivery in the TST wind tunnel. (1) Air + seeds inlet from seeding generator; (2) multiorifice distribution pipe in the settling chamber; (3) seeded air stream; (4) camera optical window and model; (5) laser sheet; (6) reflecting prism; (7) laser-light optical window

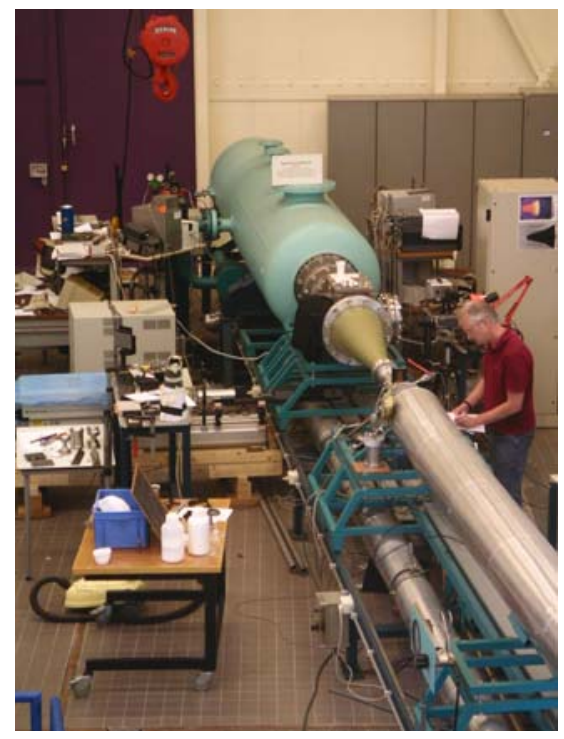

Fig. 10. Hypersonic Test Facility Delft (HTFD) with installed PIV system

due to the fact that the flow in the storage tube can be seeded offline, removing the need of a large mass flow rate through the seeder. PIV experiments in the Mach range 4 to 6 and 6 to 10 were performed in the Shock Tube of ISL (Saint Louis) and in the Hypersonic Test Facility Delft (HTFD), respectively (Figs. 10 and 11). In these conditions, the freestream can be seeded completely and rather uniformly, as shown in Figs. 12 and 13.

\section{Seeding Concentration}

The seeding concentration for compressible flows strongly reveals the flow features, which generally degrades the quality of the PIV measurement tech- 

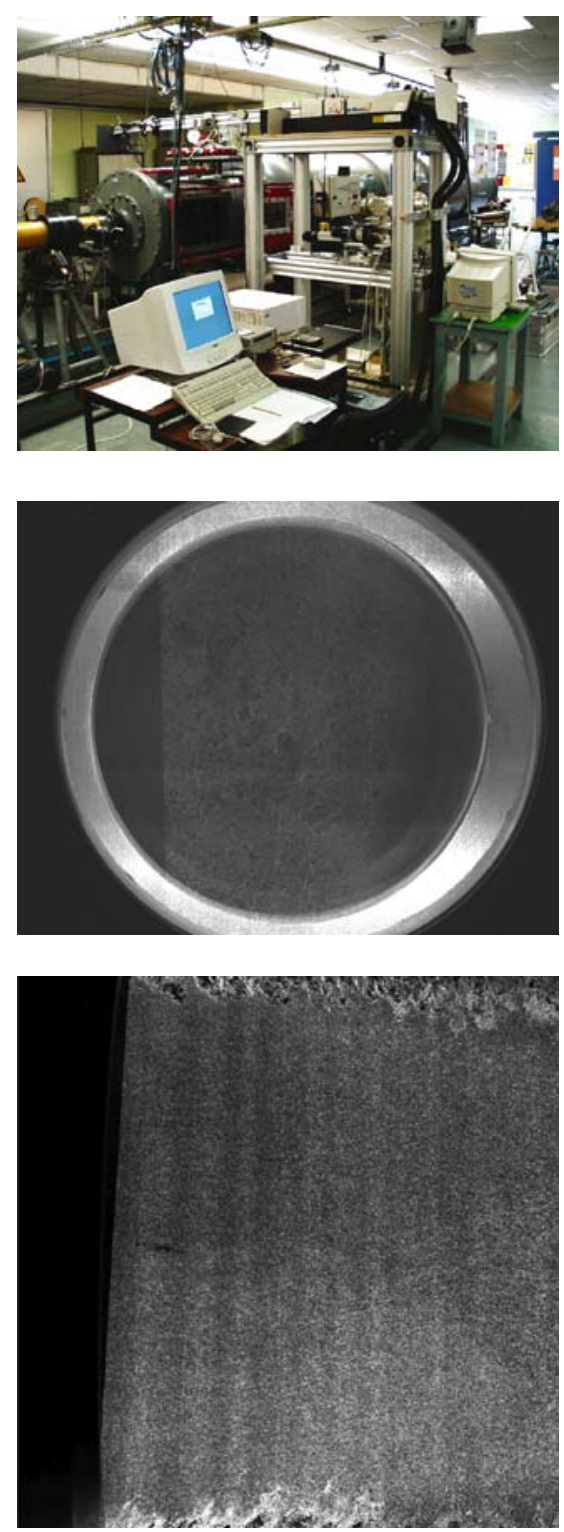

Fig. 11. ISL shock tunnel with PIV system (Haertig et al. 2002)

Fig. 12. PIV picture of $\mathrm{TiO}_{2}$ particles in HTFD at Mach 7

Fig. 13. PIV picture of $\mathrm{TiO}_{2}$ particles in ISL shock tunnel at Mach 4.5 

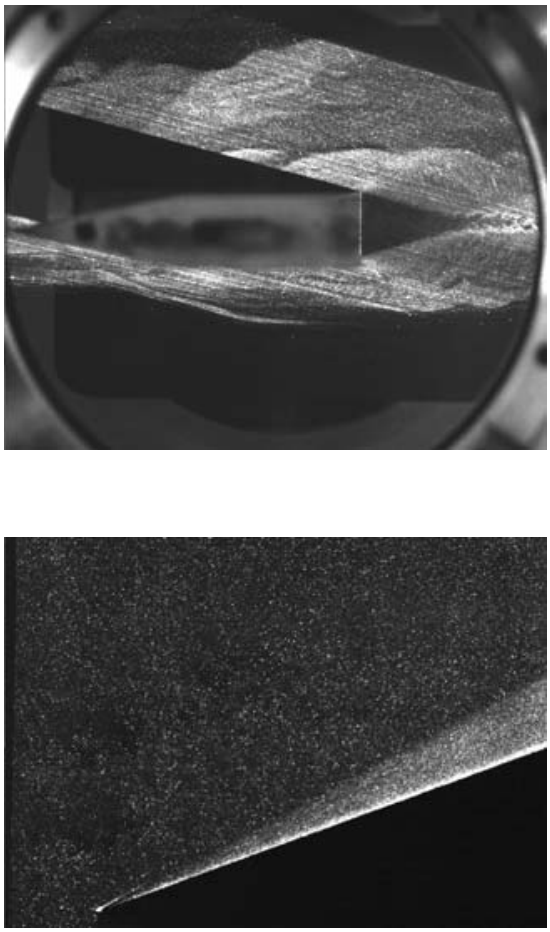

Fig. 14. Supersonic flow around a wedge-plate model in TST-27 at Mach 2

Fig. 15. Supersonic wedge flow in the ISL shock tube at Mach 6 [32]

nique. There are several causes leading to nonhomogeneous seeding concentration within high-speed flow experiments:

- inhomogeneous seeding dispersion upstream of the test section (mostly occurring for online seeding with poor mixing, Fig. 14);

- compressibility effects such as shocks, expansions and viscous layers (particle tracers concentration follows the thermodynamic density, Figs. 15 and 17); particle-tracer ejection from rotational flow regions (vortices, separated flow, boundary layers, Figs. 14 and 16).

The effects of compressibility cannot be avoided; therefore imaging devices used for supersonic flows should have a high dynamic range in order to detect a small light intensity and at the same time not saturate.

\section{Hypersonic Compression Ramp Flow}

The application of PIV in a Mach 7 flow over a two-dimensional double compression ramp is described here. The application is related to the study of atmospheric re-entry vehicles from orbital missions. The aerodynamic phenomenon of shock-shock and shock-wave-boundary-layer interaction plays an 


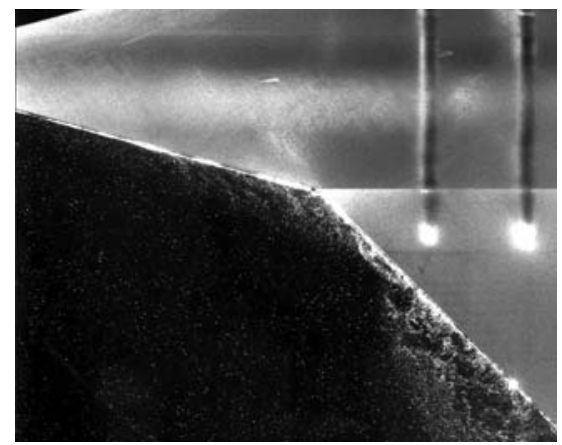

Fig. 16. Double ramp flow at Mach 7

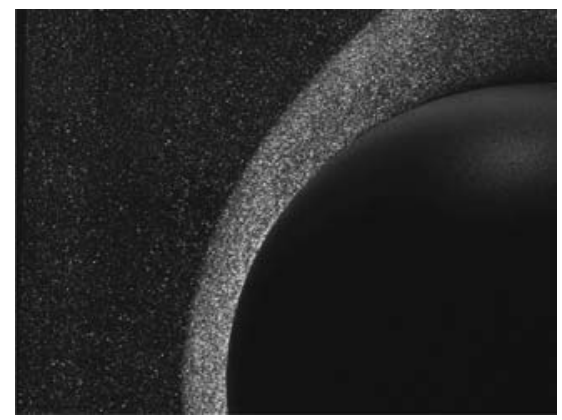

Fig. 17. Flow around a sphere at Mach 6

important role especially in the proximity of concave walls and at the insertion of control surfaces. Such interactions cause high local heat and pressure loads affecting the performance of control surfaces or the structural integrity of the vehicle. A generic representation of a control surface configuration is the 2D ramp flow, where a compression ramp induces an adverse pressure gradient triggering boundary-layer separation and a further interaction with the external flow through the separation shock. At reattachment on the ramp a system of compression waves emerges from the boundary layer, eventually coalescing into a shock [29].

The overall phenomenon is accompanied by high localized surface heat transfer, as reported in $[33,34]$.

Most studies were conducted for moderate ramp angles $\left(\alpha<30^{\circ}\right)$ where an attached straight shock solution is possible and the associated interaction pattern is relatively simple and well understood. Conversely, the flow over a ramp with $15-45^{\circ}$ deflection presents a conceptually different situation since no attached shock solution is able to realize the imposed flow deflection. Therefore, a curved shock characterizes the flow compression over the ramp and the resulting shock interaction exhibits a more complex pattern. The complexity of the phenomenon is further increased by the unsteady behavior of the separated flow region and of the shocks in the proximity of the 

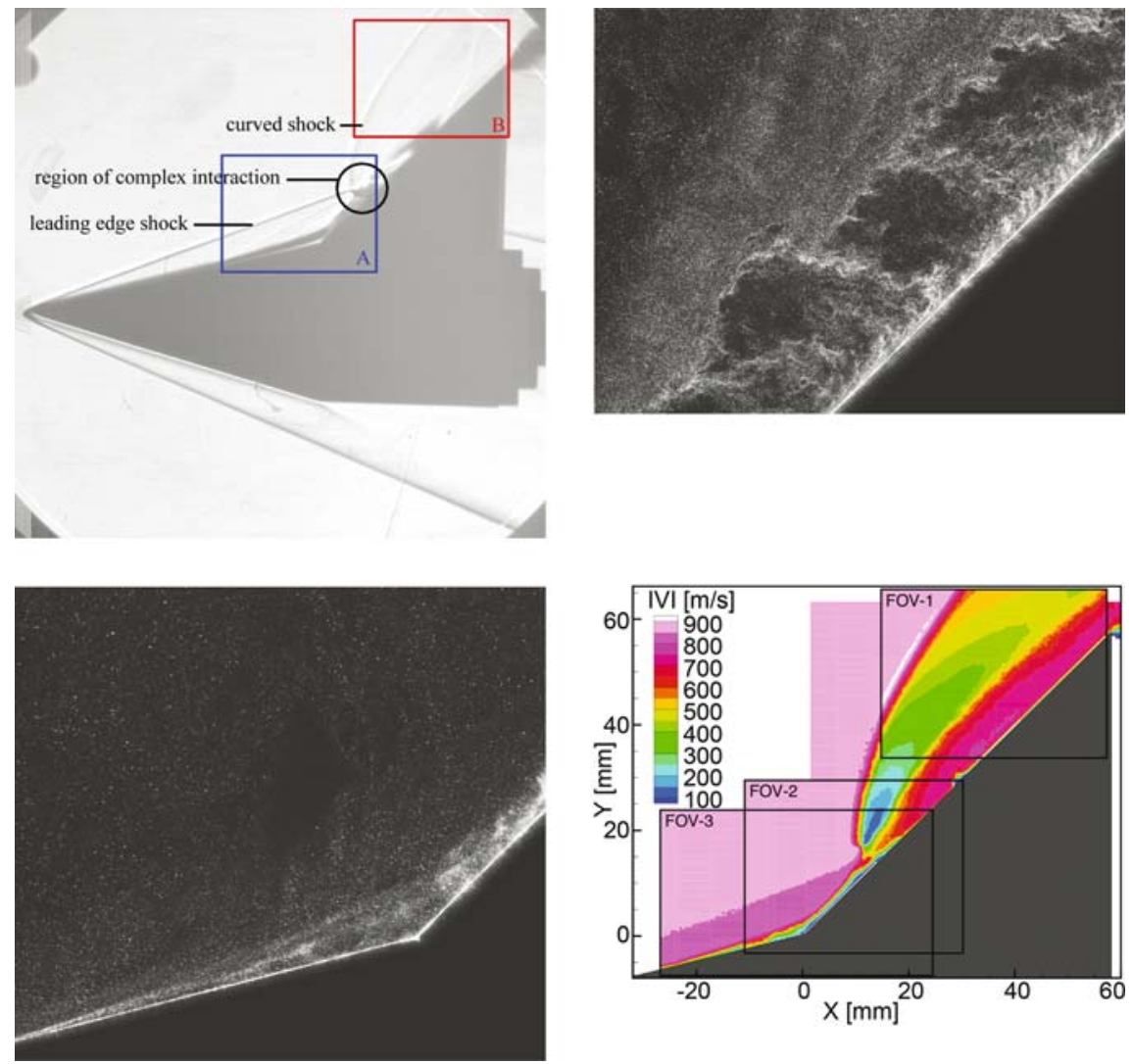

Fig. 18. Schlieren photograph of the double-ramp flow (top left); PIV recording in the separation region (bottom left); PIV recording detail downstream of the interaction (top right); combined mean velocity field (bottom right)

interaction. This difficulty also applies to numerical techniques attempting the simulation of such flow because of the large uncertainty introduced by the modeling of unsteady fluctuations by turbulence models. As a result, a considerable discrepancy has been reported between experiments and CFD results [35]. PIV experiments conducted at Mach 7 in the HTFD facility aim at the measurement of the velocity field associated to such flow phenomena. The reservoir conditions are $T_{0}=740 \mathrm{~K}$ and $P_{0}=100 \mathrm{bar}$ for temperature and pressure respectively. A fast-acting valve puts the high-pressure side in communication with a vacuum vessel at $P_{\mathrm{v}}=0.2$ mbar through a convergentdivergent axial symmetric nozzle. The test-section diameter is $D=35 \mathrm{~cm}$. The flow is seeded with $400-\mathrm{nm}$ diameter $\mathrm{TiO}_{2}$ particles illuminated by a $400 \mathrm{~J} /$ pulse Nd:YAG laser with pulse separation of $1 \mu \mathrm{s}$. The fluid velocity in the free stream is about $1040 \mathrm{~m} / \mathrm{s}$, and the particle-tracer relaxation time and length is $2 \mu \mathrm{s}$ and $2 \mathrm{~mm}$, respectively. The compressible flow features 

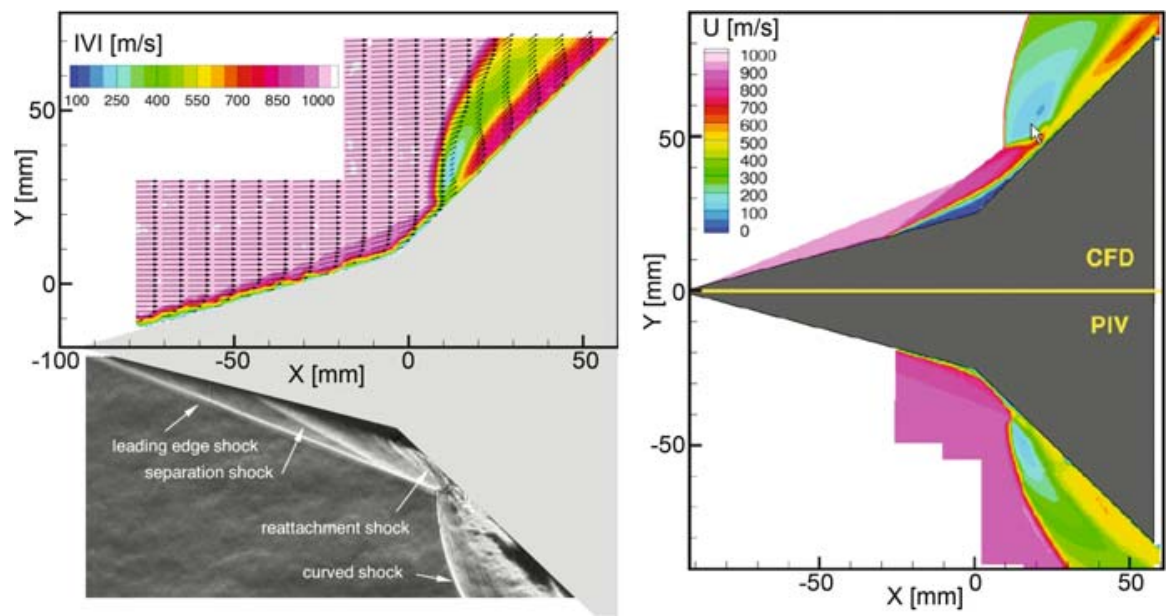

Fig. 19. Comparison of PIV and Schlieren (left, [29]. CFD results versus experiments (right)

are visualized with Schlieren and surface heat transfer is measured by means of quantitative infrared thermography (QIRT). The comparison between the Schlieren visualization and PIV shows that the overall shock pattern is captured with both techniques (Fig. 19, left), however, weak shocks cannot be clearly identified by the velocity measurement due to the limited dynamic range of single PIV snapshots. Conversely, the velocity field past the reattachment and downstream of the quasinormal shock can only be accessed by PIV with a quantitative description of the postshock flow velocity. The comparison with a 2D numerical simulation based on a Navier-Stokes solver and Menter shear stress turbulence model, returns a remarkable agreement concerning the shock pattern and the postshock velocity distribution. However, a significant discrepancy is observed concerning the extent of flow separation and its interaction with the external flow. In the CFD results, the sharp separation generates a compression shock interacting with the curved shock downstream away from the wall as opposed to the experimental results suggesting a much thinner separation and a weaker separation shock that basically merges with the leading-edge shock prior to encountering the ramp shock. This difference is also ascribed to the finite span of the wind-tunnel model opposed to the $2 \mathrm{D}$ flow simulation.

\section{Shock-Wave-Boundary-Layer Interaction}

The present section describes the experimental study by PIV of a classical problem of high-speed aerodynamics, namely the interaction between a 
fully developed turbulent boundary and a compression shock impinging on it (SWBLI). Several investigations have been reported on the subject for more than fifty years, as discussed in a review [36], yet some questions are left unanswered and predictive tools for the flow properties at the interaction are not yet completely reliable. The boundary layer developing along the upper wall of the divergent nozzle of the TST-27 wind tunnel is perturbed by a planar oblique shock wave generated by a two-dimensional wedge [21]. The wedge is placed in the free stream and imposes a flow deflection of 10 degrees. A schematic of the experimental setup is given in Fig. 20, top left. The Schlieren photograph in Fig. 20, top right, illustrates the phenomenon: a turbulent boundary layer is detected as the slightly darker corrugated region close to the wall; the impinging shock interferes with the boundary layer and is turned into a normal shock approaching the sonic line towards the wall. The effect of the adverse pressure gradient is transmitted upstream along the boundary layer and a system of compression waves is formed upstream of the impinging shock (see also Fig. 20, bottom). The unsteady nature of the flow originates from the interaction of the turbulent structures in the boundary layer and the foot of the reflected shock. Moreover, for strong interactions the wall flow between the reflected and impinging shock locally separates and turbulence fluctuations are amplified along the free shear layer producing large-scale turbulent structures. Although the Schlieren photograph allows observation of the overall pattern of the interaction, the details of the flow close to the wall require a planar quantitative measurement technique to be fully described.

The PIV recordings are analyzed with a resolution-adaptive method [37], refining the window size close to the solid wall. The windows are also stretched along the wall direction, thus maximizing the spatial resolution along the wall-normal direction. From the instantaneous velocity distribution (Fig. 21) the complex dynamics of the wall flow becomes more apparent: the turbulent boundary layer subject to the negative pressure gradient tends to separate; the shear layer lifted off the surface undergoes further instability and generates large coherent structures; the shear layer delimits a region of reverse flow at the wall. Such behavior occurs intermittently, alternating separation events with attached flow condition. The instantaneous separation seems to correlate with a locally increased shape factor for the incoming boundary layer (emptier velocity profile) associated with the local occurrence of a lowspeed streak. Conversely, for a fuller velocity profile in the incoming flow the interaction does not exhibit flow separation. Given the probability of reverse flow for this specific configuration no region of separated flow is observed in the mean flow pattern. An inflection point is observed in the velocity profile close to the wall in the region of interaction, as also reported by [21]. 


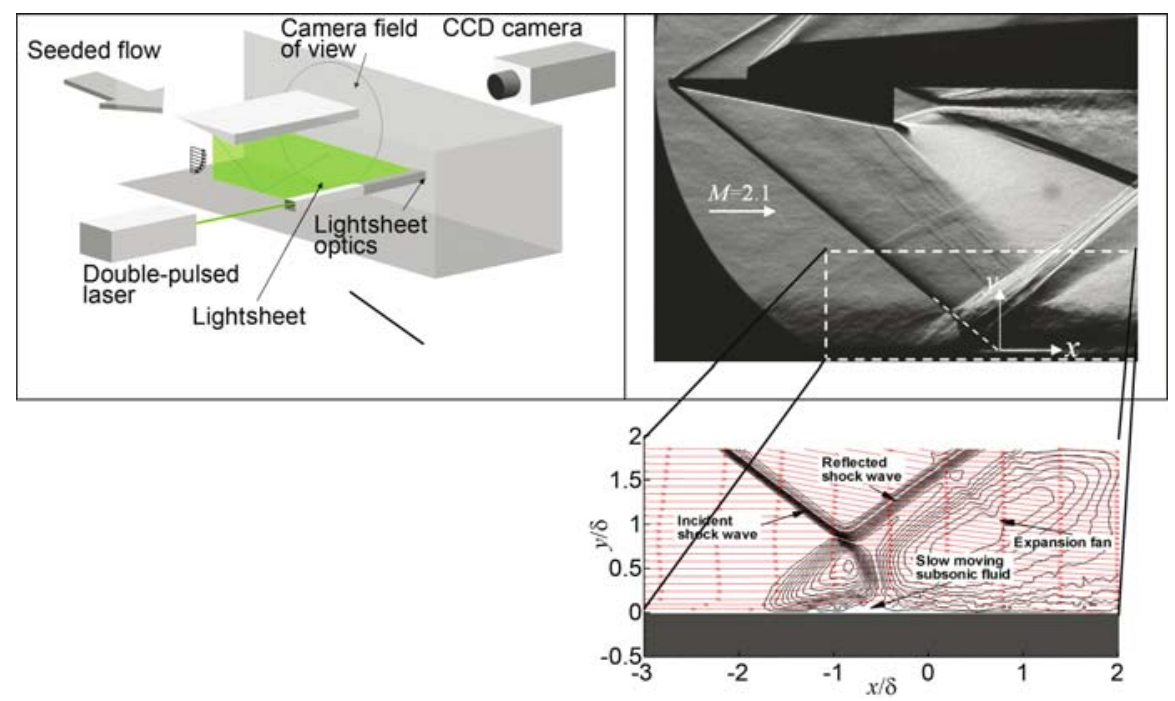

Fig. 20. Top left: SWBLI experiment with test section, illumination and imaging arrangement. Top right: Schlieren photograph (50 ns exposure time). Bottom: SWBLI flow schematic based on PIV data

\section{Conclusions}

The developments of PIV in the high-speed flow regimes result nowadays in the possibility to investigate supersonic and hypersonic flows even in the turbulent regime and for three-dimensional configurations. The physical properties of particle tracers need a careful assessment in order to limit the error due to nonideal flow tracking. The oblique shock-wave test represents an appropriate method yielding unambiguous evaluation of the particle response time, which is approximately $2 \mu \mathrm{s}$ for submicrometer solid particle tracers. The seeding distribution in high-speed facilities presents more difficulties with respect to the low-speed and especially closed-loop wind tunnels. Seeding has to be produced under pressurized conditions and transported into the settling chamber. The operation of PIV for short-duration facilities like high-pressure discharge tubes is simplified by the fact that offline seeding of the working fluid can be applied. In the hypersonic flow regime, the limiting factors for the measurement accuracy are the finite particle response and the large variations in the seeding density resulting from the large compressibility effects. Temporal separation of the exposures of less than a microsecond is often required for flow velocities of the order of $1000 \mathrm{~m} / \mathrm{s}$. The application of PIV to supersonic turbulent boundary layer flows interacting with shock waves shows that accurate mean and fluctuating velocity statistics can be obtained, which compare with the qualitative level obtained in the low-speed regime. Finally, the study of compression ramps in the hypersonic regime yields quantitative 

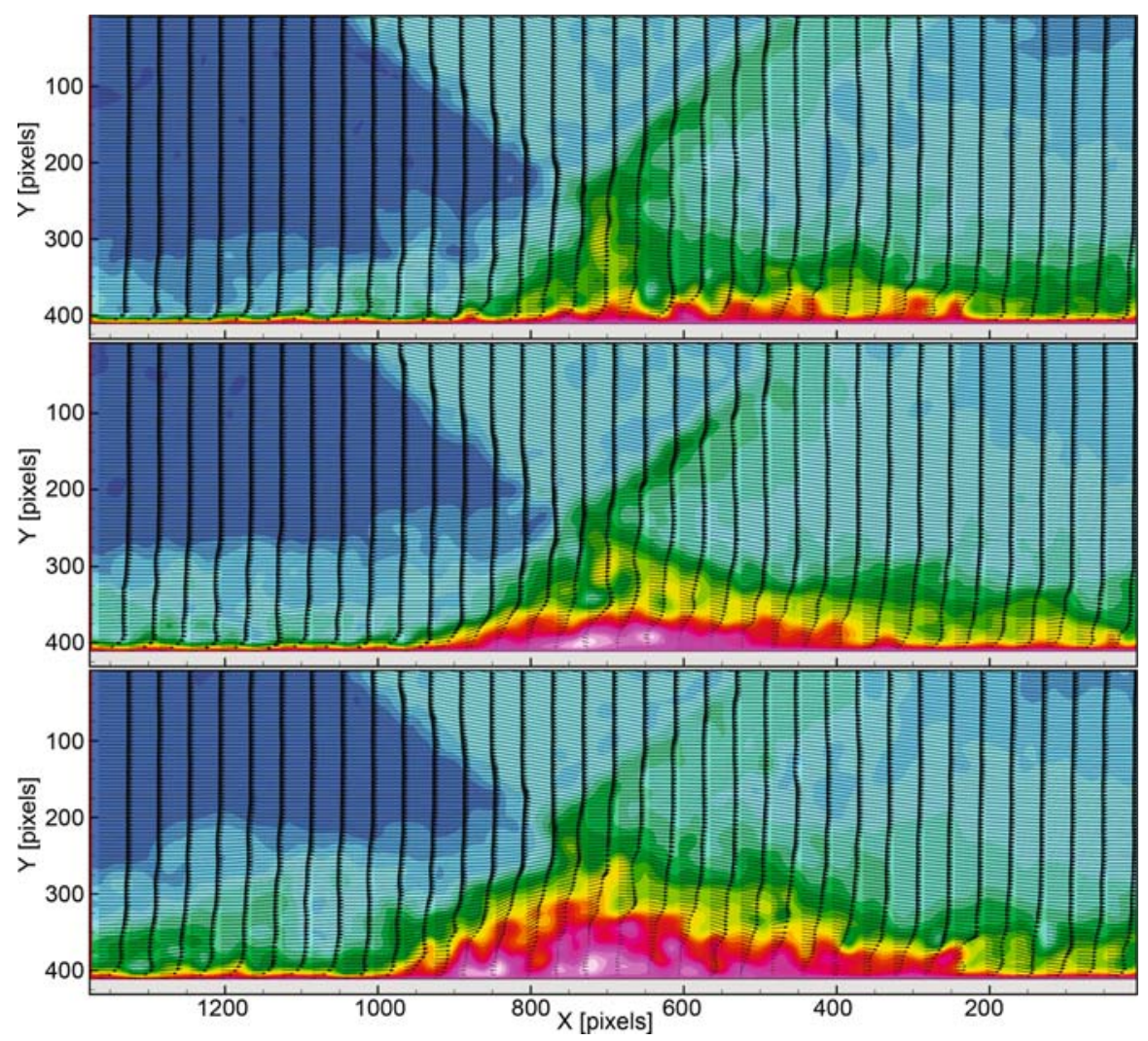

Fig. 21. Three instantaneous (uncorrelated) velocity fields. Velocity vectors and streamwise velocity contours

velocity information, which can be compared with the flow field computed by CFD with the perspective for a more extensive comparison between these means of investigation.

\section{Acknowledgements}

B. van Oudheusden, F. Schrijer, G. Elsinga, R. Humble and R. Theunissen have contributed to the research conducted at TU Delft.

This work is supported by the Dutch Technology Foundation STW under the VIDI - Innovation impulse scheme, Grant DLR.6198. 


\section{References}

[1] G. Settles: Schlieren 85 Shadowgraph Techniques (Springer, Berlin, Heidelberg 2001) 445

[2] D. Kastell, G. Eitelberg: A combined holographic interferometer and laserschlieren system applied to high temperature, high velocity flows, in Instrumentation in Aerospace Simulation Facilities (ICIASF '95 Record, International Congress 18-21 1985) pp. 12/1-12/7 445

[3] S. R. Sanderson, H. G. Hornung, B. Sturtevant: The influence of nonequilibrium dissociation on the flow produced by shock impingement on a blunt body, J. Fluid Mech. 516, 1-37 (2004) 445

[4] V. A. Amatucci, J. C. Dutton, D. W. Kuntz, A. L. Addy: Two-stream, supersonic, wake flow field behind a thick base. General features, AIAA J. 30, 2039 (1992) 445, 447

[5] R. Benay, B. Chanetz, B. Mangin, L. Vandomme, J. Perraud: Shock wave/transitional boundary-layer interactions in hypersonic flow, AIAA J. 44, 1243-1254 (2006) 445

[6] N. T. Clemens, M. G. Mungal: A planar Mie scattering technique for visualizing supersonic mixing flows, Exp. Fluids 11, 175 (1991) 445

[7] N. T. Clemens, M. G. Mungal: Large scale structure and entrainment in the supersonic mixing layer, J. Fluid Mech. 284, 171 (1995) 445

[8] R. J. Adrian: Twenty years of particle image velocimetry, in 12th Int. Symp. Appl. of Laser Tech. Fluid Mech. (2004) 445

[9] A. J. Smits, J. P. Dussauge: Turbulent Shear Layers in Supersonic Flow (AIP Press 1996) 446

[10] G. E. Elsinga, B. W. van Oudheusden, F. Scarano: Evaluation of optical distortion in PIV, Exp. Fluids 39 (2005) 446

[11] C. S. Moraitis, M. L. Riethmuller: Particle image displacement velocimetry applied in high speed flows, in Proc. 4th Int. Symp. Appl. of Laser Anemometry to Fluid Dyn. (1988) 446

[12] J. Kompenhans, R. Höcker: Application of particle image velocimetry to high speed flows, in Riethmuller, M. L. (Eds.): Particle image displacement velocimetry, VKI Lecture Series (1988) 446

[13] A. Krothapalli, D. P. Wishart, L. M. Lourenço: Near field structure of a supersonic jet: 'on-line' PIV study, in Proc. 7th Int. Symp. Appl. of Laser Tech. Fluid Mech. (1994) 446

[14] Lourenço, L. M.: Particle image velocimetry, in M. L. Riethmuller (Ed.): Particle Image Velocimetry, VKI Lecture Series 1996-03 (1996) 446

[15] M. Raffel, H. Höfer, F. Kost, C. Willert, J. Kompenhans: Experimental aspects of PIV measurements of transonic flow fields at a trailing edge model of a turbine blade, in Proc. 8th Int. Symp. Appl. of Laser Tech. Fluid Mech. (1996) paper No. 28.1.1 446

[16] M. P. Wernet: Digital PIV measurements in the diffuser of a high speed centrifugal compressor, in (1998) AIAA Paper No. 98-2777 446

[17] W. D. Urban, M. G. P. Mungal: Velocity measurements in compressible mixing layers, J. Fluid Mech. 431, 189 (2001) 446, 447, 450

[18] F. Scarano, B. W. Van Oudheusden: Planar velocity measurements of a twodimensional compressible wake, Exp. Fluids 34, 430-441 (2003) 446, 447, 450 
[19] F. Scarano: PIV image analysis for compressible turbulent flows. Extension of PIV to the hypersonic flow regime, particle tracers assessment, in Lecture Series on Advanced measuring techniques for Supersonic Flows (von Kármán Institute for Fluid Dynamics, Rhode-Saint Genèse 2005) 446

[20] F. Scarano: Quantitative flow visualization in the high speed regime: Heritage, current trends and perspectives, in Proc. 12th Int. Symp. On Flow Vis. (2006) paper No. 2.1446

[21] R. A. Humble, F. Scarano, B. W. van Oudheusden, M. Tuinstra: PIV measurements of a shock wave/turbulent boundary layer interaction, in Proc. 13th Int. Symp. Appl. of Laser Tech. Fluid Mech. (2006) 446, 458

[22] J. Haertig, M. Havermann, C. Rey, A. George: Particle image velocimetry in Mach 3.5 and 4.5 shock-tunnel flows, AIAA J. 40, 1056 (2002) 446

[23] F. F. J. Schrijer, F. Scarano, B. W. van Oudheusden: Application of PIV in a hypersonic double-ramp flow, in (2005) AIAA paper No. 2005-3331 446

[24] A. Melling: Tracer particles and seeding for particle image velocimetry, Meas. Sci. Technol. 8, 1406 (1997) 446, 447

[25] G. Tedeschi, H. Gouin, M. Elena: Motion of tracer particles in supersonic flows, Exp. Fluids 26, 288 (1999) 446

[26] S. A. Schaaf, P. L. Chambre: Fundamentals of Gas Dynamics (Princeton Univ. Press 1958) 446

[27] J. L. Herrin, J. C. Dutton: Effect of a rapid expansion on the development of compressible free shear layers, Phys. Fluids 7, 159 (1995) 447

[28] M. Samimy, S. K. Lele: Motion of particles with inertia in a compressible free shear layer, Phys. Fluids A 3, 1915 (1991) 447

[29] F. F. J. Schrijer, F. Scarano, B. W. van Oudheusden: Application of PIV in a Mach 7 double-ramp flow, Exp. Fluids 41 (2006) 447, 455, 457

[30] M. Raffel, C. Willert, J. Kompenhans: Particle Image Velocimetry, a Practical Guide (Springer, New York 1988) 447, 448, 450

[31] G. E. Elsinga, B. W. van Oudheusden, F. Scarano: The effect of particle image blur on the correlation map and velocity measurement in PIV, in Optical Engineering and Instrumentation, SPIE Annual Meeting (2005) 448

[32] M. Havermann, J. Haertig, C. Rey, A. George: Application of particle image velocimetry to high-speed supersonic flows in a shock tunnel, in 11th Int. Symp. on Applications of Laser Techniques to Fluid Mechanics, (2002) 454

[33] W. L. Hankey, Jr, M. S. Holden: Two-dimensional shock-wave boundary layer interactions in high speed flows, in AGARDograph, vol. 203 (1975) 455

[34] J. Délery, J. G. Marvin: Shock-wave boundary layer interactions, in AG 280, AGARD (1986) 455

[35] K. Sinha, M. J. Wright, G. V. Candler: The effect of turbulence on double-cone shock interactions, in (1999) AIAA paper No. 99-0146 456

[36] D. S. Dolling: Fifty years of shock wave/boundary layer interation research: What next?, AIAA J. 39 (2001) 458

[37] R. Theunissen, F. F. J. Schrijer, F. Scarano, M. L. Riethmuller: Application of adaptive PIV interrogation in a hypersonic flow, in Proc. 13th Int. Symp. Appl. of Laser Tech. Fluid Mech. (2006) 458 


\section{Index}

boundary-layer separation, 455

compressibility effect, 454

compressible flow, 445

compression ramp, 454

curved shock, 455

cyclone separator, 451

drag relation, 446

hypersonic wind tunnel, 451

hypersonic wind tunnel flow, 445

optical distortion, 448

particle

agglomeration, 447

particle image velocimetry, 445

particle relaxation time, 446, 447 particle response, 447

particle tracers slip, 445

particle-image blur, 445

quantitative infrared thermography, 457

shock wave, 445

shock-wave turbulent boundary interaction, 445

shock-wave-boundary-layer interaction, 454,457

slip velocity, 447

supersonic, 445

temporal resolution, 448

titanium dioxide, 451

turbulence model, 457

turbulent mixing, 446 